\title{
Atmospheric water vapour content over La Silla Paranal Observatory
}

\author{
R. Querel ${ }^{1}$, F. Kerber ${ }^{2}$, R. Hanuschik ${ }^{2}$, G. Lo Curto ${ }^{3}$, \\ D. Naylor ${ }^{1}$, M. Sarazin ${ }^{2}$ and A. Smette ${ }^{3}$ \\ ${ }^{1}$ Institute for Space Imaging Science, University of Lethbridge, Lethbridge, AB, Canada \\ ${ }^{2}$ European Southern Observatory, Garching, Germany \\ ${ }^{3}$ European Southern Observatory, Santiago, Chile
}

Water vapour is the principle source of opacity at infrared wavelengths in the earth's atmosphere. Measurements of atmospheric water vapour serve two primary purposes when considering operation of an observatory: long-term monitoring of precipital water vapour (PWV) is useful for characterizing potential observatory sites, and real-time monitoring of PWV is useful for optimizing use, in particular for mid-IR observations.

In support of site testing for the European Extremely Large Telescope (E-ELT), we have used La Silla and Paranal as calibration sites to ground-truth satellite measurements of PWV. To this end, dedicated measurement campaigns have been conducted over both sites and Las Campanas Observatory ( $\mathrm{LCO}$ ) through a collaboration between the University of Lethbridge, scientists from the European Southern Observatory, and the Giant Magellan Telescope site test team at LCO.

Several independent measurement techniques were used in this study. Continuous measurements were obtained using IRMA infrared radiometers at $20 \mu \mathrm{m}$. PWV was also retrieved from spectra covering the wavelength range from the visible to the infrared using facility instruments (FEROS, HARPS and MIKE, UVES, CRIRES, VISIR) through fitting their data to simulated atmospheric spectra. A prototype 3-band lunar spectrophotometer, operating at $\sim 0.94 \mu \mathrm{m}$, was also employed.

Local meteorological data were provided by a series of radiosonde launches timed to coincide with satellite overpasses. The radiosondes provided in situ measurements of PWV, and time and location specific atmospheric profiles. Together, this multi-faceted approach has resulted in a unique data set. Integral to this analysis is a site specific atmospheric radiative transfer model (BTRAM), common to all retrieval schemes.

We also have reconstructed the PWV history over the La Silla Paranal observatory, by analysing $>2000$ high-resolution echelle spectra of spectrophotometric standard stars using archival FEROS and UVES data. The extracted PWV values were compared to ENVISAT and GOES satellites to allow a statistical analysis of PWV over several years.

\section{Acknowledgements}

We would like to acknowledge Greg Tompkins for his electronics expertise; the AstroMeteorology Group at the Universidad de Valparaiso for launching the radiosondes; and Joanna Thomas-Osip and Gabriel Prieto (Las Campanas) for excellent collaboration. D.N. acknowledges support from NSERC, AIF and CFI.

\section{References}

Thomas-Osip, J., McWilliam, A., Phillips, M. M. et al., 2007, PASP, 119, 697

Querel, R. R., Naylor, D. A., Thomas-Osip, J. et al., 2008, SPIE, 7014E.172Q 